\title{
A new high efficiency organic inhibitor applied to prevent coal spontaneous combustion
}

\author{
Genyin CHENG $^{1, a}$, Fei CHEN ${ }^{2, b}$,Yaqin JIANG ${ }^{2, c}$ Ming GAO $^{2, d}$ \\ ${ }^{1}$ Occupation Safety and Health Cooperative Innovation Center, North China University of Science \\ and Technology, Box 206, Yanjiao Beijing 101601, China \\ ${ }^{2}$ School of Environmental Engineering, North China University of Science and Technology, Box \\ 206, Yanjiao Beijing 101601, China \\ a 1298275966@qq.com,, 291032156@qq.com, c 2224399257@ qq.com, d gaoming@ncist.edu.cn
}

Keywords: inhibitor; cone calorimeter; coal; spontaneous combustion

\begin{abstract}
A new high efficiency organic inhibitor (HEOI) was synthesized, which was applied to coal to inhibit the spontaneous combustion. Meanwhile, $\mathrm{MgCl}_{2}$ was used to compare the inhibition properties with HEOI, studied by cone calorimeter. It was found that the combustion parameters of coal samples, including total heat release (THR), carbon monoxide release, and mass loss were decreased considerably by the treatment. Moreover, for coal sample treated with HEOI, the decrease was obviously more than that of coal sample treated with $\mathrm{MgCl}_{2}$. For total amount of smoke release, the coal sample treated with HEOI was much higher than that of coal sample treated with $\mathrm{MgCl}_{2}$. We come to a conclusion that HEOI has a better effect on preventing coal spontaneous combustion.
\end{abstract}

\section{Introduction}

In china, coal is the basic energy of our country, which occupies about $75 \%$ in primary energy, and it is in the leading position in the of energy consumption. However, spontaneous combustion of coal causes serious accident and economic losses in the process of the storage and transportation of coal ${ }^{[1]}$. Coal spontaneous combustion caused huge economic losses, casualties, and seriously pollution. It has great importance to prevent coal spontaneous combustion.

Nowadays, along with the progress of modern science and technology and the research on the mechanism of coal spontaneous combustion, all kinds of fire prevention and control measures of coal spontaneous combustion are progressively established. There are a lot of inhibitors on the market, the conventional inhibitors include halogen salt, ammonium salt, some chemical inhibitors, and so on ${ }^{[2-3]}$.

Salt blocking agent, the source is widespread, the craft is simple, the resistance of the effect is good, but the endurance is poor ${ }^{[4]}$. Ammonium salt can absorb the heat generated by coal spontaneous combustion. Its resistant efficiency is not high, and the resistance of the process is to extend the ignition time, which means that ammonium salt ${ }^{[5]}$ did not really stop the burning of coal. Alkali resistance agent, low cost, but due to the small solubility, it is easy to plug, which seriously affects the resistance effect, in addition, alkali resistance agent has high material requirements of the equipment due to its strong corrosiveness. The gel blocking agent is formed by the action of the base material and the accelerating agent in a certain proportion. This kind inhibitor is expected to play an important role in the field of fire-fighting technology in coal mine. But silica gel inhibition agent glue process releases a large number of ammonia, which causes certain hazards on the human body. Other gel resistance of agent or viscosity, the resistance of the effect is not ideal, the relatively high cost or the preparation process are too complex, cumbersome procedures, and some other defects.

So it is necessary to synthesize a new type of highly efficient organic inhibitor, which has low cost, wide application range, long service life, non-toxic and high efficiency characters. 


\section{Experimental}

\section{Materials.}

Dicyandiamide $\left(\mathrm{C}_{2} \mathrm{H}_{4} \mathrm{~N}_{4}\right.$; analytical reagent), phosphoric acid $\left(\mathrm{H}_{3} \mathrm{PO}_{4} ; 85 \%\right)$ and formaldehyde (HCHO; 37\%) were supplied by Tianjin Yongda Chemical Reagent Co. Anhydrous magnesium chloride $\left(\mathrm{MgCl}_{2}\right.$; analytical reagent) and clay were supplied by Shenzhen Kesheng Trading Company ltd. Coal (coal Long flame coal; 0.125-0.180mm) was supplied by Inner Mongolia coal mine.

\section{Measurements and characterization}

Cone calorimeter (PX-07-007; Feinikesi quality control instrument Company Limited), the specimen size for the cone calorimetry experiments was $10 \times 10 \times 30 \mathrm{~mm}^{3}$. At least three samples were tested to obtain average values ${ }^{[6]}$.

\section{Synthesis of the inhibitor.}

The reaction principle is as follows:

(1) $\mathrm{H}_{2} \mathrm{NC}(\mathrm{NH})_{2} \mathrm{CN}+\mathrm{H}_{3} \mathrm{PO}_{4}+\mathrm{H}_{2} \mathrm{O} \rightarrow \mathrm{H}_{2} \mathrm{NC}(\mathrm{NH})_{2} \mathrm{C}(\mathrm{O}) \mathrm{NH}_{2} \cdot \mathrm{H}_{3} \mathrm{PO}_{4}$

(2) $\mathrm{H}_{2} \mathrm{NC}(\mathrm{NH})_{2} \mathrm{C}(\mathrm{O}) \mathrm{NH} \cdot \mathrm{H}_{3} \mathrm{PO}_{4}+\mathrm{HCHO} \rightarrow \mathrm{HOCH}_{2} \mathrm{NHC}(\mathrm{NH})_{2} \mathrm{C}(\mathrm{O}) \mathrm{NH}_{2} \cdot \mathrm{H}_{3} \mathrm{PO}_{4}$

$50 \mathrm{~g}$ of dicyandiamide and water $80 \mathrm{~mL}$ of water were added to $200 \mathrm{~mL}$ three neck bottle, the resulting mixture was heated to 90 to $100^{\circ} \mathrm{C}$ to dissolve dicyandiamide. $40 \mathrm{~mL}$ of phosphoric acid (85\%) was added in the mixing process, the mixture was heated to $100^{\circ} \mathrm{C}$ and hold at the temperature for approximately 40 min to complete. The mixture was cooled to $80^{\circ} \mathrm{C} .51 \mathrm{~mL}$ of formaldehyde (37\%) was added to the three neck bottle, then the temperature of mixture was kept at $80^{\circ} \mathrm{C}$ for $30 \sim 40 \mathrm{~min}$. The reaction is completed when the solution viscosity increased, the blocking agent with solid content of about $51.42 \%$ is obtained, which is the new high efficiency organic inhibitor (HEOI).

\section{Screening of coal sample}

In this experiment, we choose the long flame coal of Inner Mongolia coal, the calorific value is 21. $42 \mathrm{MJ} / \mathrm{kg}$, the sulfur content in the long flame coal is $0.495 \%$, and its spontaneous combustion period is about $10-12$ months, the size of coal sample used in this experiment is $0.125-0.180 \mathrm{~mm}$.

\section{Preparation of coal sample}

Firstly, $180 \mathrm{~mL}$ of different concentrations of the inhibitor and the preparation of $20 \%$ magnesium chloride are prepared. Then, 5 coal samples and each $45 \mathrm{~g}$ were set out, and the size of the coal samples is between $0.125 \mathrm{~mm}-0.180 \mathrm{~mm}$. The five coal samples were placed in five $250 \mathrm{ml}$ beaker, 20\% $\mathrm{MgCl}_{2}$, 50\% HEOI, 30\% HEOI, 20\% HEOI and 15\% HEOI were also added to the five beakers, then a blender was used to mix the mixture respectively, and it will last for an hour. The samples were drying for 4 hours after Standing for 12 hours. So we got the coal samples treated with the inhibitor at different concentrations.

\section{Cone calorimeter test analysis}

Coal samples were texted by cone calorimeter, such as raw coal, coal sample treated with $20 \%$ $\mathrm{MgCl}_{2}$ and HEOI at different concentrations. Total heat release, carbon monoxide (CO) release, mass loss, total smoke release are used to analyze the effects of inhibitors, which is applied to prevent coal spontaneous combustion. 


\section{Results and discussion}

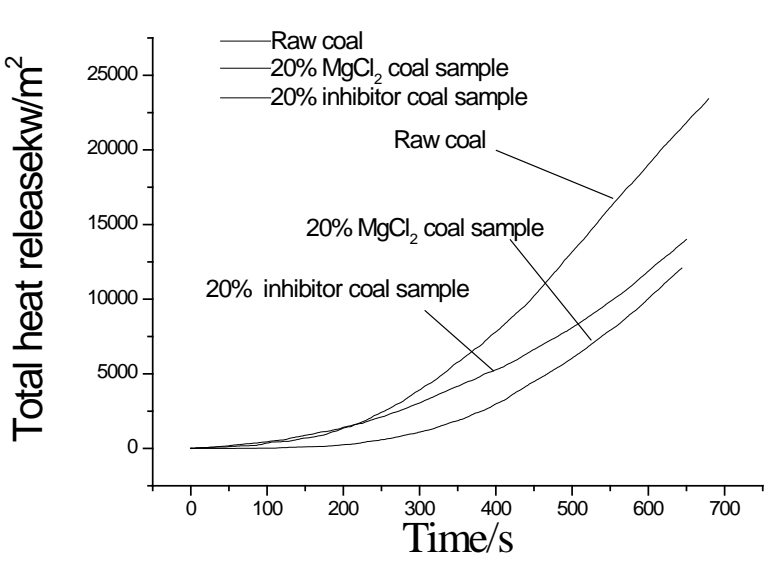

Figure1. Total heat release of coal samples

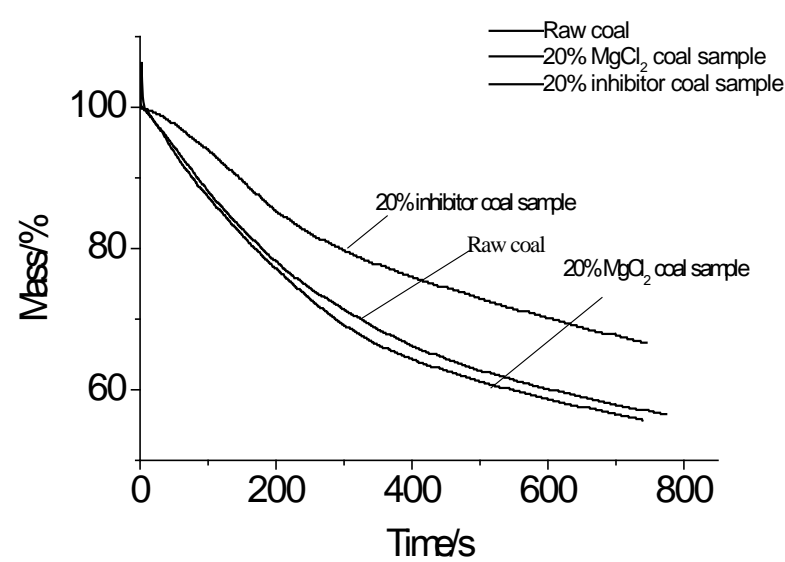

Figure3. Mass fraction of coal samples

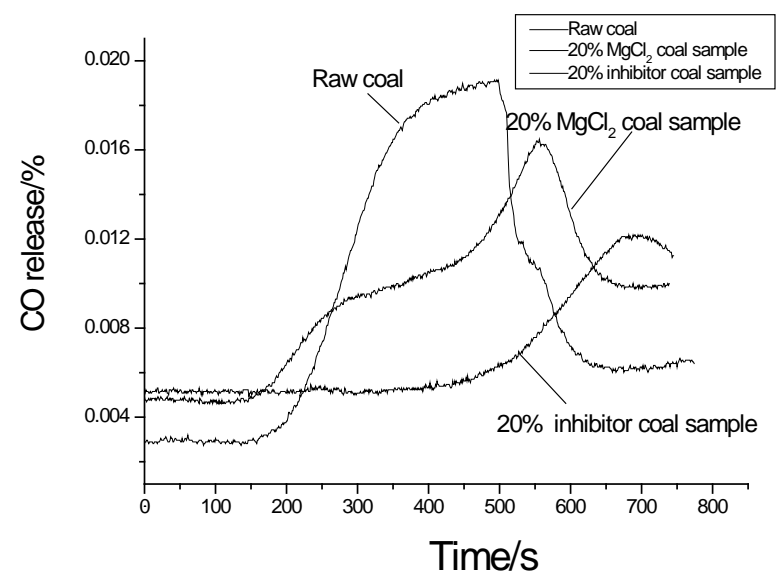

Figure2. CO release of coal samples

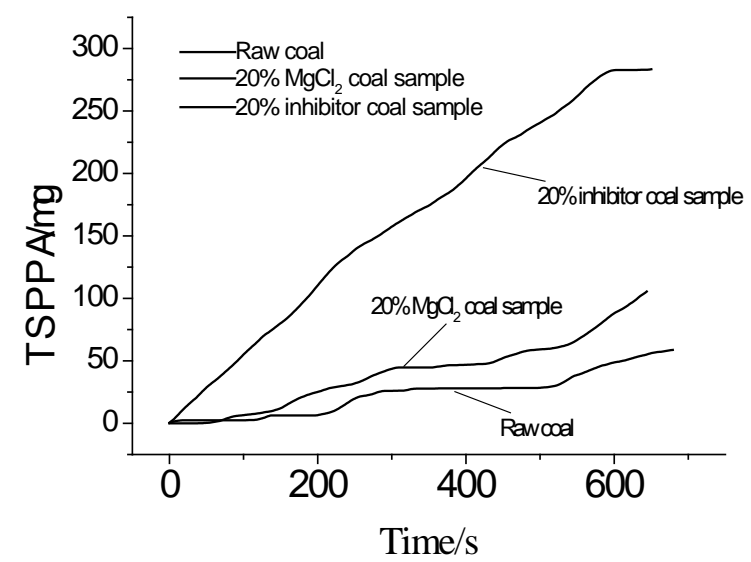

Figure4. Total smoke release of coal samples

\section{Total heat release analysis}

Figure 1 shows the heat release of coal samples changes along with the time. It can be clearly found that, at the beginning, the heat release of three coal samples tend to rise slowly, and the gap of the heat release began to widen after 350s. In the 650s, the heat release of raw coal reach the maximum, the amount of heat release from the coal sample treated with $20 \% \mathrm{MgCl}_{2}$ followed, and the heat release of coal sample treated with $20 \%$ HEOI is lowest. This shows that HEOI formed a layer of insulation film on the surface of coal sample, makes the heat produced from coal is hard to release, which made the heat release lowest among the three coal samples. $\mathrm{MgCl}_{2}$ also has certain effect on the heat release of coal sample, but the persistence of $\mathrm{MgCl}_{2}$ is poor than HEOI, and its inhibition effect is slightly deficient. Meanwhile, the new high efficiency organic inhibitor (HEOI) has a quite well effect on the spontaneous combustion of coal.

\section{Carbon monoxide release analysis}

Figure 2 shows the amount of carbon monoxide release of different coal samples change with time. From the figure 2, the release of carbon monoxide from three kinds of coals is certain in the 0-150s, almost unchanged. Starting from 200s, the carbon monoxide begins to release, the coal samples combusted incompletely at this point, raw coal sample release carbon monoxide fast, $20 \%$ 
$\mathrm{MgCl}_{2}$ coal sample followed, the release of carbon monoxide from the coal sample treated with $20 \%$ HEOI is the slowest. When the time comes to 500s, the amount of carbon dioxide emissions from raw coal reached the maximum, meanwhile, the coal sample treated by $20 \%$ HEOI start releasing carbon monoxide. After 500s, the amount of carbon monoxide released from the raw coal began to decrease rapidly, which showed that the raw coal sample began to enter the complete combustion state. In the 580s, the carbon monoxide released from the coal sample treated with $20 \%$ $\mathrm{MgCl}_{2}$ begin to decrease, and the coal sample treated by $20 \%$ HEOI start to reduce the rate of release of carbon monoxide in the 700s. This phenomenon fully indicated that HEOI has a function to inhibitor the release of carbon monoxide when the coal is combusting. HEOI has an inhibitory effect on the release of toxic gases from coal. Indirectly, it is indicated that HEOI has a great influence on the spontaneous combustion of coal, and the inhibitor effect is also very good.

\section{Mass loss analysis}

As is shown in Figure 3, the mass fraction of different coal samples varies with time. And from the Figure 3, it can be seen that the quality of the coal sample treated with $20 \% \mathrm{MgCl}_{2}$ decreases more quickly compared with the raw coal sample and the coal sample treated with 20\% HEOI. However, the decrease in weight of the coal sample treated with 20\% HEOI is slower than the other two coal samples. Compared with the mass decrease of raw coal sample, the coal sample treated with $20 \% \mathrm{MgCl}_{2}$ decrease more, which shows that the $20 \% \mathrm{MgCl}_{2}$ does not have good effect on the suppression of the burning of coal samples in high temperature conditions. Meanwhile, the quality of the coal sample treated with 20\%HEOI decreased most slowly among the three coal samples, which means 20\%HEOI has excellent effect on prevent coal spontaneous combustion in high temperature conditions ${ }^{[7]}$. Therefore, the new high efficiency organic inhibitor has a good inhibitory effect, and its inhibitory effect is better than that of $20 \% \mathrm{MgCl}_{2}$.

\section{Total amount of smoke release}

Figure 4 indicates that the amount of smoke release of different coals varies over time. As is can be seen from the figure 4, at the beginning of the coal sample treated with $20 \%$ HEOI began to release smoke, it is a great amount of release, basically a straight rise. Conversely, the curve is very smooth in raw coal smoke release, the coal combusted very completely. Compared with the smoke release of the coal samples treated with $20 \% \mathrm{HEOI}$ and $20 \% \mathrm{MgCl}_{2}$, the raw coal sample's is very little, basically no smoke release from raw coal sample. We take the amount of smoke release of the coal samples treated with $20 \%$ HEOI and $20 \% \mathrm{MgCl}_{2}$ into consideration that coal sample treated with 20\% HEOI released more smoke, which indicates that 20\% HEOI can be used to replace 20\% $\mathrm{MgCl}_{2}$ when it comes to prevent coal spontaneous combustion. As we can see from the Figure 4, the amount release of $20 \% \mathrm{HEOI} / \mathrm{coal}$ sample has reached $300 \mathrm{mg}$ in 600s, and the raw coal was released less than $50 \mathrm{mg}$ of the smoke, this gap obviously increases. In the coal oxidation, the accumulation of temperature will not cause the burning of coal samples, even if the coal has been oxidized, because of the blocking agent on the surface of coal has formed a good oxygen denial of membrane, it will inhibit the binding of coal and oxygen to prevent the coal self heating and combustion.

\section{Conclusions}

$20 \%$ HEOI form can form a very good protective film on the surface of coal, the membrane can block coal combustion, making coal sample heat can not release or release less. The release of carbon monoxide of coal can be inhibited. Compared with the resistance of $\mathrm{MgCl}_{2}$ to the coal sample, the resistance of HEOI is even better. The mass loss of coal sample treated with HEOI is much lower than that of coal sample treated with $\mathrm{MgCl}_{2}$.

In summary: through experiments, we can completely replace the inorganic inhibitor in the ordinary sense with the new high efficiency organic inhibitor, which can be applied to the fire 
prevention in the coal mine well.

\section{Acknowledgement}

The work was supported by joint fund of the national Natural Foundation (U1361130) and the fundamental research funds for the Central Universities (3142015021)

\section{References}

[1] LC Li, ZY Zheng, QY Wang. Polyethylene as a novel low-temperature inhibitor for lignite coal. Journal of Thermal Analysis \& Calorimetry, 2014, 117(3):1321-1325

[2] Wu J, Yang J, NakagoshiN, LuX,XuH. Research on technology for preventing spontaneous combustion of coal. Adv Mater Res.2012; 524-527:677-80.

[3] Pandey J, Mohalik NK, Mishra RK, Khalkho A, Kumar D et al. Investigation of the role of fire retardants in preventing sponta-neous heating of coal and controlling coal mine fires. Fire Technol. 2012; $1-19$.

[4] Taraba B, Peter R, Slova'k V. Calorimetric investigation of chemical additives affecting oxidation of coal at low temperatures. Fuel Process Technol. 2011; 92(3):712-5.

[5]Wu K, Wang Z, Hu Y. Microencapsulated ammonium poly-phosphate with urea-melamine-formaldehyde shell: preparation,characterization, and its flame retardance in polypropylene. Polym Advan Technol. 2008;19(8):1118-25.

[6] W Li, C Han, W Liu, M Zhang, K Tao. Expanded graphite applied in the catalytic process as a catalyst support. Catalysis Today, 2007, 125(3):278-281

[7] L Zhang, GQ Zhu, GW Zhang, RS Han. Performance-based Fire Design of Air-supported Membrane Coal Storage Shed. Procedia Engineering. 2013, 52:593-601 